\title{
PREFERENCIAS ENTRE CINCO CARBOHIDRATOS EN PHEIDOLE BILIMEKI (HYMENOPTERA: FORMICIDAE)
}

\author{
Madai ROSAS-MEJÍA, ${ }^{1}$ Alfonso CORREA-SANDOVAL, ${ }^{2}$ Crystian SAdiel VENEGAS-BARRERA ${ }^{3}$ y Jorge \\ VÍCTOR HORTA-VEGA ${ }^{4}$
}

\begin{abstract}
${ }^{1}$ Instituto de Ecología Aplicada, Universidad Autónoma de Tamaulipas, Avenida División del Golfo No 356, La Libertad, Ciudad Victoria, Tamaulipas, México, C. P. 87019.<mrosasmejia@yahoo.com.mx>.

${ }^{2-4}$ Instituto Tecnológico de Cd. Victoria. Blvd. Emilio Portes Gil 1301 Pte. Cd. Victoria, Tamaulipas, México. C.P. 87010. ${ }^{2}$ <agutierr@uat.edu.mx>, ${ }^{3}<$ crystianv@gmail.com>, ${ }^{4}<$ jhortavega@yahoo.com.mx>.
\end{abstract}

Recibido: 21/01/2015; aceptado: 09/06/2015

Rosas-Mejía, M., Correa-Sandoval, A., Venegas-Barrera, C. S. \& Horta-Vega, J. V. 2015. Preferencias entre cinco carbohidratos en Pheidole bilimeki (Hymenoptera: Formicidae). Acta Zoológica Mexicana (n. s.), 31(2): 291-297.

RESUMEN. Los carbohidratos comunes en fuentes naturales de energía para las hormigas son: sacarosa, fructosa, glucosa, trehalosa y melezitosa. En este estudio se establecieron preferencias entre estos cinco carbohidratos en Pheidole bilimeki Mayr a través de la conducta de reclutamiento. El orden de efectividad reclutadora de los cinco químicos fue: sacarosa $>$ melezitosa $=$ trehalosa $>$ fructosa $>$ glucosa. En todos los azúcares la respuesta fue dependiente de la concentración entre $0.1 \mathrm{M}$ y $1 \mathrm{M}$. Los tiempos bebiendo fueron los mismos en todos los carbohidratos, por lo cual se descarta que distintos volúmenes de solución ingerida determinen diferentes cantidades de feromona reclutadora secretada. Se propone que diferentes niveles de interacción de los azúcares con los receptores gustativos determina la intensidad del reflejo liberador de la feromona.

Palabras clave: hormigas, trehalosa, feromona reclutadora, melezitosa.

\section{INTRODUCCIÓN}

La conducta de reclutamiento en las hormigas es el proceso de comunicación que convoca a individuos de una colonia a dirigirse hacia algún sitio en donde el trabajo es requerido (Wilson 1971). Cuando se trata de transportar suministros energéticos, las obreras forrajeras después de localizar e ingerir provechosamente un alimento regresan al nido liberando una feromona a manera de rastro olfativo, con esto estimulan a otras hormigas para trasladarse hacia el recurso (Carthy 1950; Wilson 1959; Hölldobler \& Wilson 1990; Detrain \& Deneubourg 1997). La conducta de reclutamiento depende, entre otros factores, del tipo de alimento (Cammaerts \& Cammaerts 1980; Breed et al. 1987; Horta-Vega et al. 2010), la calidad (Mailleux et al. 2000; Tinti \& Nofre 2001) y alternativas de elección (Kay 2004; Horta-Vega et al. 2010; Wilder \& Eubanks 2010). El objetivo del presente es evaluar la respuesta de reclutamiento de la especie generalista Pheidole bilimeki Mayr ante cinco tipos de carbohidratos comunes en la naturaleza: plantas, secreciones de áfidos y hemolinfa de
Rosas-Mejía, M., Correa-Sandoval, A., Venegas-Barrera, C. S. \& Horta-Vega, J. V. 2015. Preferences among five carbohydrates in Pheidole bilimeki (Hymenoptera: Formicidae). Acta Zoológica Mexicana (n. s.), 31(2): 291-297.

ABSTRACT. Sucrose, fructose, glucose, trehalose and melezitose are sugars commonly found in natural sources of energy of ants. In this study were established preferences through the recruitment behavior to these carbohydrates in Pheidole bilimeki Mayr. The order of effectiveness of the five chemicals was: sucrose $>$ melezitose $=$ trehalose $>$ fructose $>$ glucose. In all sugars the responses were dependent to the concentration between $0.1 \mathrm{M}$ and $1 \mathrm{M}$. The time invested in drinking per ant was the same in all carbohydrates, therefore, it is discarded that different volumes of solution ingested could determine different amounts of secreted trail pheromone. We propose that different levels of interaction of sugars with their gustatory receptors could determine the intensity of pheromone releasing reflex.

Key words: ants, trehalose, pheromone recruitment, melezitose.

insectos. Se aborda el factor de calidad y la exposición dual simultánea para evaluar preferencias.

\section{MATERIAL Y MÉTODOS}

Área de estudio. El trabajo se realizó en un área ubicada dentro del campus del Instituto Tecnológico de Cd. Victoria, Tamaulipas, México (2345'21.24'’N; 99¹0'02.95' 'O; altitud: 325 msnm), se trabajó con nidos localizados en el suelo de Pheidole bilimeki entre los meses de junio y septiembre de 2014. La especie es de las más abundantes cuando se colectan hormigas con trampas pitfall en la zona urbana del municipio (datos no publicados). Los experimentos se realizaron entre las 9:00-12:00 y 16:00-19:00 horas evitando el periodo de mayor insolación y procurando siempre mantener los nidos sombreados durante los procedimientos. La temperatura ambiente promedio a lo largo del trabajo fue de $35.2{ }^{\circ} \mathrm{C}$, frecuente en esta época en la localidad (datos proporcionados por la Comisión Nacional del Agua, 2014). 
Carbohidratos y registro del reclutamiento. Los carbohidratos utilizados fueron sacarosa, $\mathrm{D}(-)$ fructosa, $\mathrm{D}(+)$ glucosa, $\mathrm{D}(+)$ trehalosa y $\mathrm{D}(+)$ melezitosa, todas de la marca SIGMA ${ }^{\circledR}$. Los carbohidratos se presentaron en viales cilíndricos de plástico de color blanco, de $1 \mathrm{~cm}$ de alto y $1.8 \mathrm{~cm}$ de diámetro, con capacidad de $2 \mathrm{~mL}$. La actividad reclutadora fue determinada por el número de individuos que se encontraban bebiendo en el tiempo establecido en cada experimento. El conteo se realizó a partir de imágenes digitales adquiridas con una cámara Canon ${ }^{\circledR}$ EOS Rebel XT (Fig. 1) o con una videocámara Canon ${ }^{\circledR}$ Vixia HF20, con la cual también se determinaron los tiempos de ingesta invertidos por las hormigas.

Respuesta a cada carbohidrato. Para evaluar el poder fagoestimulante reclutador de cada químico por separado, se colocaron sobre el suelo 60 viales al azar durante 90 min en un área de $20 \times 20 \mathrm{~m}$ con presencia de la hormiga. El día 1 se inició con sacarosa y durante los siguientes días cada uno de los otros azúcares, todos a una concentración $0.5 \mathrm{M}$. El testigo consistió en viales con agua destilada. La estrategia además permitió la localización puntual de la entrada de los nidos para las pruebas posteriores con diferentes concentraciones y en los experimentos sobre preferencias entre los carbohidratos. En estas pruebas, y antes de iniciar cada experimento, se colocaron cebos de embutido de carne (chorizo de pavo) cerca de varios de los nidos para determinar en cuáles de ellos trabajar de acuerdo a la presencia de actividad exploradora (HortaVega et al. 2010). Los protocolos arrancaron después de 60 min de retirado el cebo. Para el cálculo del promedio de la respuesta de reclutamiento se consideraron solo aquellos viales con al menos 10 individuos, así se descartan hormigas arribando aleatoriamente.

Sensibilidad a los carbohidratos. Para determinar que la concentración de $0.5 \mathrm{M}$ no satura la respuesta fagoestimulante se realizó una comparación de respuesta de reclutamiento de las hormigas bilimeki en los cinco carbohidratos a concentraciones de $0.1 \mathrm{M}, 0.5 \mathrm{M}$ y $1.0 \mathrm{M}$ cada uno, bajo el mismo procedimiento de respuesta a cada carbohidrato. La respuesta se cuantificó a los 60 minutos de exposición del carbohidrato.

Dinámica de reclutamiento. El curso temporal del reclutamiento se determinó cuantificando cada 10 minutos el número de hormigas bebiendo en los viales en un pe-

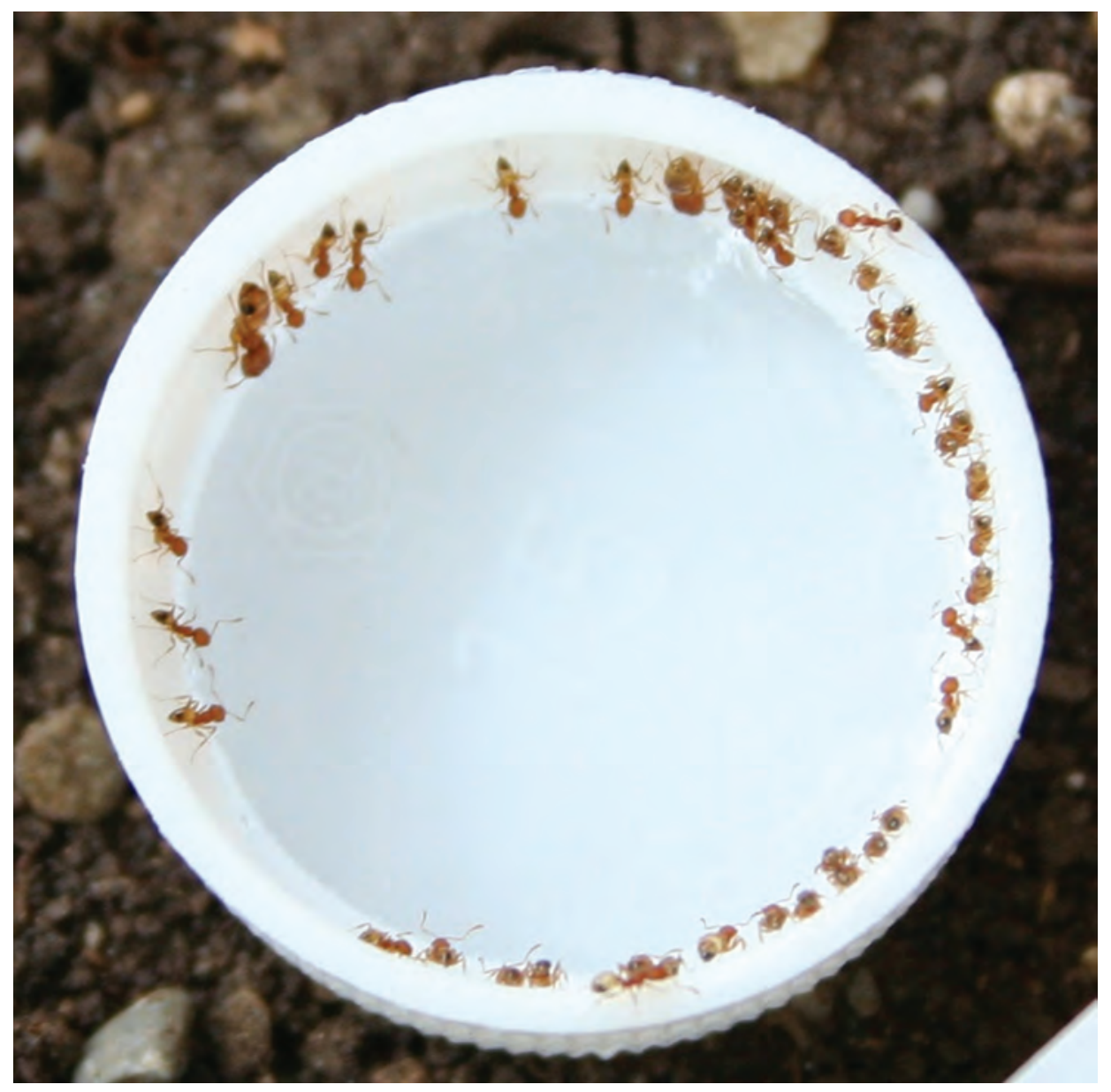

Figura 1. Fotografía de un vial con hormigas reclutadas. 
ríodo de 90 minutos. Se realizaron 7 experimentos correspondientes a 5 de sacarosa, uno de trehalosa y uno de melezitosa. Los promedios se calcularon con los valores normalizados de cada experimento.

Preferencia entre carbohidratos. Las preferencias entre los azúcares se establecieron a través de la respuesta reclutadora con la presentación simultánea de dos carbohidratos a la vez. En este protocolo y con el objeto de que las hormigas sólo tuvieran un camino de acceso a los químicos, cada par de viales con un distinto azúcar era colocado en soportes en forma de $\mathrm{T}$ con la sección vertical terminada en punta para fijarla al suelo. La altura libre del soporte sobre el suelo fue de $3 \mathrm{~cm}$ y de $6 \times 2.5$ $\mathrm{cm}$ la sección transversal superior donde se colocaban las soluciones. Los dos viales, separados $0.5 \mathrm{~cm}$ uno del otro, se intercambiaron de posición en seis ocasiones durante los primeros 30 min de un total de 90 min de exposición de los carbohidratos. Esta maniobra se implementó para evitar el establecimiento de rastros de feromona hacia el primer químico aleatoriamente encontrado. En todos los protocolos el tiempo cero correspondió a la llegada de la primera hormiga exploradora y que hizo contacto con la solución por al menos 20 segundos. El número de experimentos en cada situación fue entre cinco y diez, de donde se obtuvieron los promedios. Las comparaciones fueron la sacarosa con cada uno de los otros cuatro carbohidratos, la trehalosa con la fructosa y melezitosa, y fructosa con la glucosa.

Tiempo bebiendo. En experimentos separados se colocaron viales $(n=4)$ de cada fuente de energía o carbohidrato para cuantificar el tiempo que invierte cada una de 25 hormigas bilimeki cuando se posan a beber en los viales. La concentración fue de $0.5 \mathrm{M}$ de cada uno de los carbohidratos y además sacarosa $1.0 \mathrm{M}$ para fines de comparación. Se calculó el promedio de los tiempos bebiendo en la muestra $(n=25)$.

Análisis de datos. El análisis estadístico para la determinación de diferencias significativas comprendió a la prueba $t$ de student en el caso de comparación entre dos medias y una ANOVA de una vía para el caso de la confrontación de tres o más conjuntos de datos. Valores de $p$ menores a 0.05 fue el criterio considerado para la significancia estadística. Todos los datos sometidos al análisis provinieron de pruebas experimentales realizados en al menos tres nidos distintos.

\section{RESULTADOS}

Respuesta a cada carbohidrato. El número total de hormigas observadas en los cinco carbohidratos durante el período de estudio fue de 5363 individuos. En sacarosa se contaron 1476, le siguió melezitosa con 1089, fructosa con 939, glucosa con 934 y trehalosa con 925 hormigas. En los viales testigo con agua destilada fueron 31 individuos en total. La cantidad de hormigas en cada vial osciló entre cero y 135 individuos, es decir, algunos sitios con un nulo reclutamiento o casi nulo. La respuesta promedio de reclutamiento en cada azúcar fue: sacarosa 43.4, fructosa 27.8, glucosa 27.6, trehalosa 26.3 y melezitosa 31.5 (Fig. 2). El análisis estadístico reveló que en promedio la sacarosa presentó un efecto fagoestimulante mayor que la fructosa, glucosa y trehalosa. Con melezitosa, sin embargo, aunque también el reclutamiento fue menor en promedio que con sacarosa aquí no se observaron diferencias estadísticas significativas entre ambos azúcares. Asimismo, el análisis estadístico comparativo entre fructosa, glucosa, trehalosa y melezitosa no mostró diferencias significativas en su capacidad reclutadora (Fig. 2).

Sensibilidad a los carbohidratos. Los cinco carbohidratos probados generaron conducta reclutadora dependiente de la concentración (Fig. 3). En todos los casos se encontraron diferencias estadísticas significativas $(p<0.05)$ entre las concentraciones. Estos resultados indican que es posible distinguir la respuesta reclutadora de las colonias ante fuentes de alimento de diferente calidad a través del número de hormigas en los viales. Asimismo, los datos de la Figura 3 validan el uso de la concentración $0.5 \mathrm{M}$ para determinar preferencias entre los azúcares probados en este estudio, en función de que en ninguno de ellos a dicha concentración se obtuvo algún efecto saturante en la actividad reclutadora.

Dinámica de reclutamiento. La Figura 4 muestra el ritmo de incorporación de los individuos hacia la fuente de alimento. En la curva se observa un incremento con el tiempo hasta alcanzar un nivel asintótico después de los 60 minutos. Estos datos permitieron definir el tiempo que

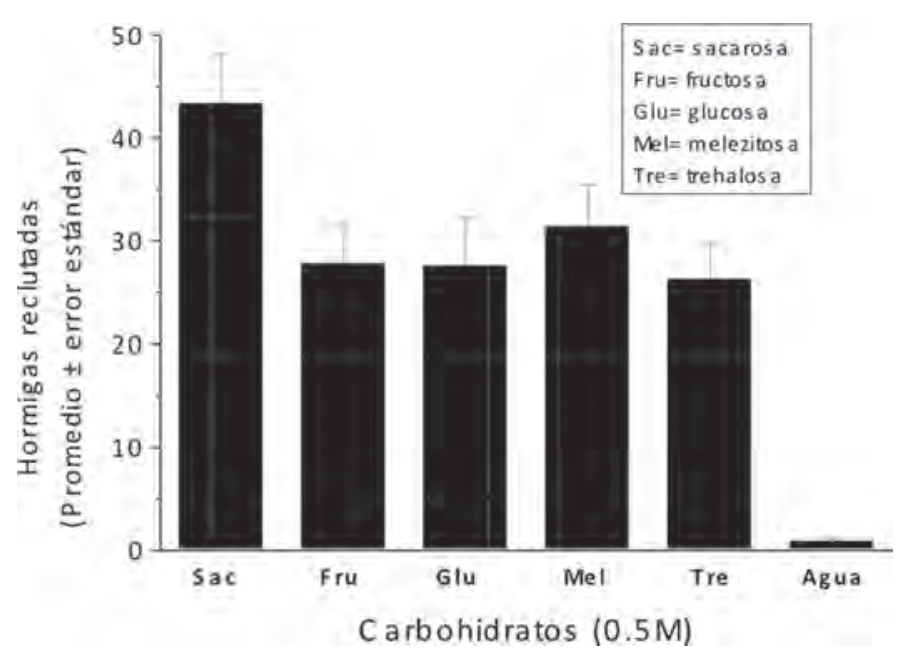

Figura 2. Número de hormigas bilimeki reclutadas en cada uno de los cinco carbohidratos y agua después de $90 \mathrm{~min}$. 


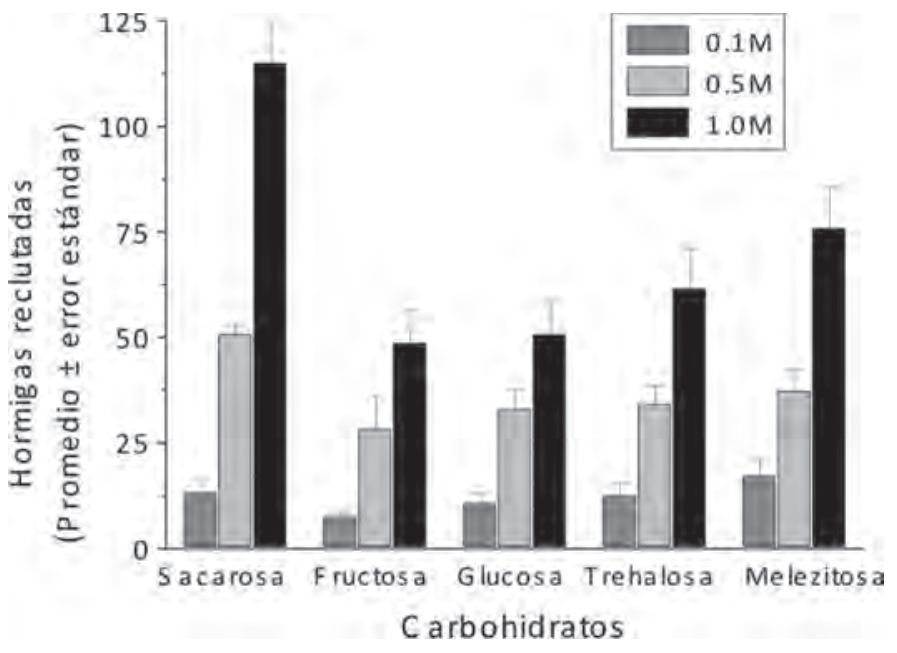

Figura 3. Reclutamiento de las hormigas bilimeki dependiente de la concentración de cinco carbohidratos.

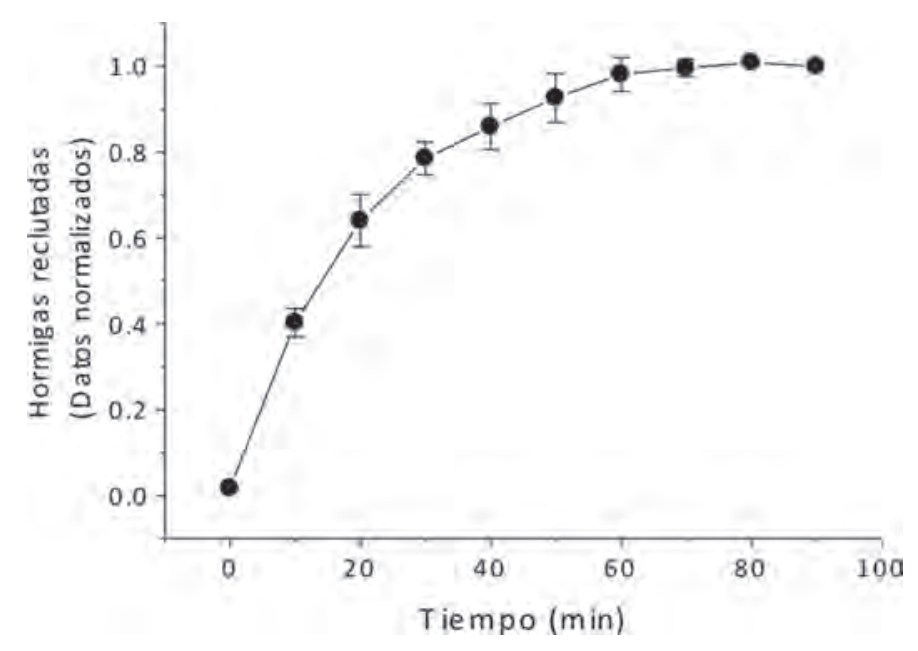

Figura 4. Curso temporal del reclutamiento de hormigas bilimeki.

invierten las colonias en llegar a un estado máximo estacionario de actividad reclutadora, y por lo tanto el tiempo óptimo para realizar comparaciones entre químicos en un estado estable de la respuesta del nido ante el hallazgo de una fuente de alimento.

Preferencias entre carbohidratos. En el número de hormigas reclutadas cuando la colonia tiene la opción de dos distintas fuentes de energía se observó que los carbohidratos glucosa, fructosa, trehalosa y melezitosa perdieron la competencia en su capacidad para reclutar en presencia de sacarosa (Fig. 5). Esto fue notoriamente significativo con glucosa y fructosa en donde en promedio se contaron un $8.5 \%$ y $13.5 \%$, respectivamente, en relación con las hormigas ubicadas en el vial de sacarosa. La trehalosa y melezitosa le dieron más competencia reclutando en promedio un $32.5 \%$ y $42.5 \%$, respectivamente, de aquéllos reclutadas por sacarosa, pero también las diferencias fueron estadísticamente significativas.

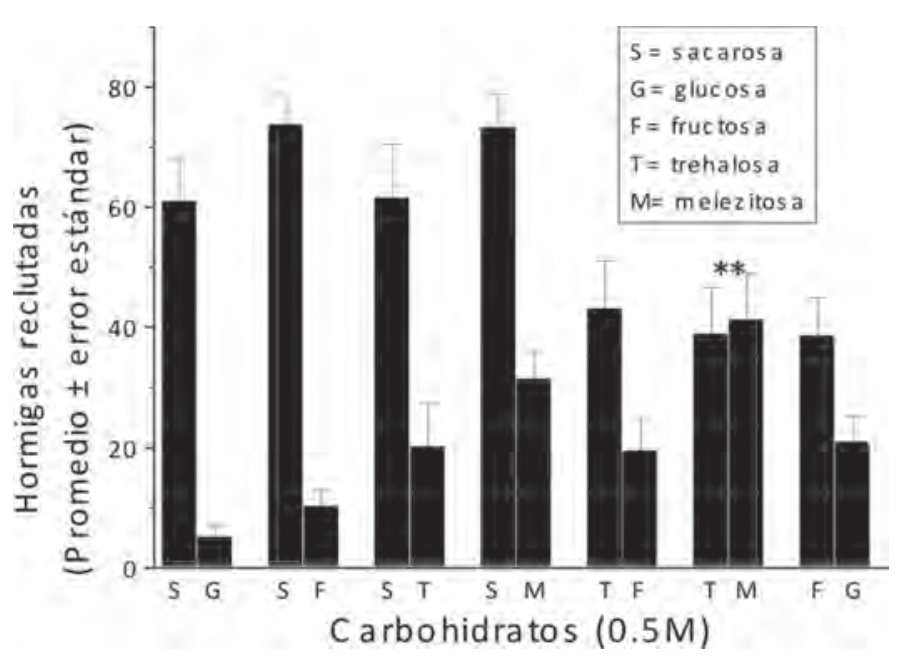

Figura 5. Preferencia entre carbohidratos en experimentos de presentación dual simultánea.

En la competencia entre los otros azúcares, la fructosa mostró un poder fagoestimulante reclutador 1.85 veces significativamente mayor en promedio que la glucosa. Pero, no obstante su predominio reclutador sobre ese monosacárido, la fructosa apenas alcanzó un $45.2 \%$ del poder estimulante registrado para la azúcar trehalosa cuando se presentaron ambos simultáneamente. En el caso de trehalosa y melezitosa, no se observó ninguna preferencia entre ambos azúcares (Fig. 5). Esta serie de combinaciones de presencia simultánea nos permiten establecer el orden de preferencia que tiene Pheidole bilimeki sobre los cinco carbohidratos: sacarosa $>$ melezitosa $=$ trehalosa $>$ fructo sa $>$ glucosa. Este es el primer reporte sobre reclutamiento y preferencias de carbohidratos en Pheidole bilimeki.

Tiempo bebiendo. Las hormigas cuando arriban a los viales hacen contacto en la solución con sus partes bucales y prácticamente sin interrupciones beben con un visible aumento (en el volumen del gáster) antes de retirarse de la solución y dirigirse hacia al nido. El tiempo que invierten las hormigas bebiendo al llegar a la fuente de energía se presenta en la Figura 6. Como se puede observar, en los cinco carbohidratos cada individuo en promedio ocupa en esencia el mismo tiempo de saciedad, entre los 73-78 segundos a la concentración $0.5 \mathrm{M}$. Sin embargo, cuando se colocó una concentración doblemente mayor como fue con sacarosa $1 \mathrm{M}$, el tiempo invertido se incrementó significativamente hasta alcanzar 101 segundos en promedio (Fig. 6).

\section{DISCUSIÓN}

En este estudio se muestran evidencias de una respuesta de reclutamiento diferencial de Pheidole bilimeki a la estimulación gustativa por carbohidratos que son comunes 


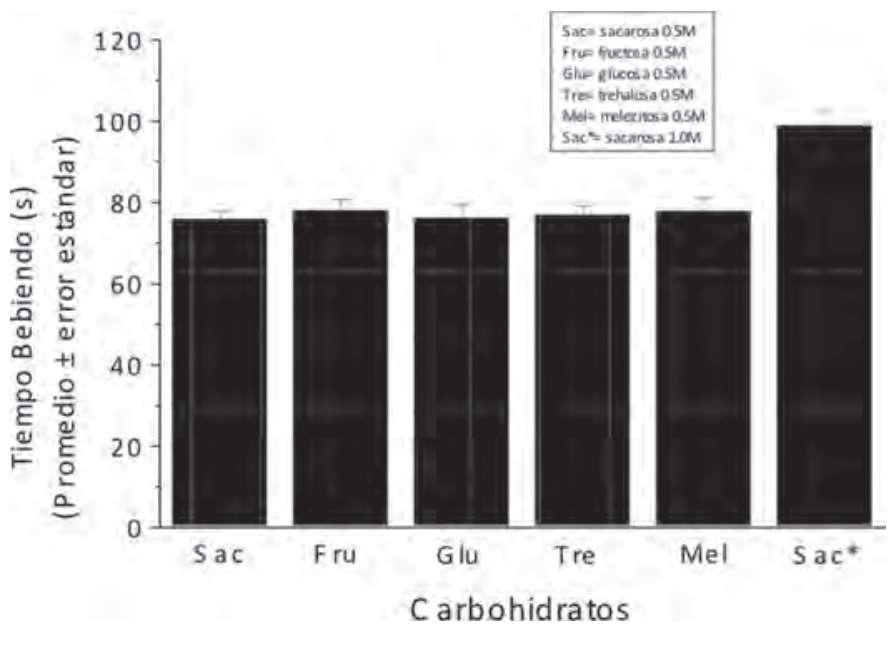

Figura 6. Tiempo bebiendo de hormigas bilimeki.

en fuentes nutricionales de origen floral, extrafloral o proveniente de áfidos (Hendrix et al. 1992; Blüthgen et al. 2004; Heil et al. 2005; Wilder \& Eubanks 2010). Distintas sensibilidades fagoestimulantes entre carbohidratos se conocen también en otras especies de hormigas (VanderMeer et al. 1995; Völkl et al. 1999; Tinti \& Nofre 2001; Blüthgen \& Fiedler 2004; Heil et al. 2005; Horta-Vega et al. 2010).

Cuando las hormigas forrajeras localizan sacarosa generan un reclutamiento mayor a lo observado con fructosa, glucosa y trehalosa, pero no con melezitosa, al menos no significativamente. En los experimentos duales, con opción simultáneamente entre dos azúcares, la preferencia sobre la sacarosa se pronuncia marcadamente, incluso hasta la melezitosa es significativamente superada. Esto sugiere que las hormigas logran diferenciar finamente el valor energético de cada carbohidrato y definir una preferencia para un mayor rendimiento del esfuerzo invertido. Una conclusión similar se describe para Pheidole dentigula (Horta-Vega et al. 2010).

La preferencia por sacarosa sobre los monosacáridos glucosa y fructosa ocurre en diversas especies de hormigas (Vander-Meer et al. 1995; Tinti \& Nofre 2001; Blüthgen \& Fiedler 2004), sin embargo, en otras especies la fructosa y glucosa tienen el mismo nivel de fagoestimulación o incluso mayor que la sacarosa (Koptur \& Truong 1998; Heil et al. 2005; Horta-Vega et al. 2010). La invertasa está difundida ampliamente entre las hormigas (Ayre 1967; Ricks \& Vinson 1970, 1972) lo cual les permite ingerir la sacarosa como fuente de energía, pero diferencias en la actividad de la enzima en cada especie podría determinar, al menos parcialmente, una mayor o menor preferencia por el disacárido sacarosa (Heil et al. 2005).

El disacárido trehalosa y el trisacárido melezitosa presentaron entre ellos un similar nivel fagoestimulante, am- bos superaron a la fructosa, pero no compitieron al mismo nivel a la sacarosa. La preferencia de trehalosa sobre fructosa contrasta con lo descrito para la hormiga Lasius niger L. (Hymenoptera: Formicidae), la cual responde bien a la fructosa pero presenta una nula o muy baja reacción a trehalosa (Tinti \& Nofre 2001; Detrain \& Prieur 2014). La trehalosa es el azúcar de mayor concentración en la hemolinfa de los insectos (Friedman 1985), se sintetiza en el cuerpo graso y es la principal fuente de energía metabólica a través de la enzima trehalasa (Becker et al. 1996). Para algunas especies su ingesta podría no ser indispensable como con Lasius niger que son visitadoras asiduas de colonias de áfidos (Hemiptera: Aphididae) (Kiss 1981), que por sus secreciones son una fuente segura de otros carbohidratos. En el caso de melezitosa, algunas especies la prefieren sobre la sacarosa (Völkl et al. 1999; Tinti \& Nofre 2001) lo cual podría explicarse por la dependencia energética de la secreciones ricas en melezitosa provenientes de insectos hemípteros de la familia Aphididae (Vantaux et al. 2011). Sin embargo, estas altas concentraciones del trisacárido en tales secreciones no siempre ocurre (Blüthgen et al. 2004). Existen evidencias de que el contenido de melezitosa puede ser altamente variable entre colonias de áfidos a través de ajustes fisiológicos de la osmolaridad para evitar la pérdida de agua en el tubo digestivo (Douglas 2003). Es frecuente que el floema de donde se alimentan los áfidos tenga una presión osmótica mayor que la hemolinfa (Douglas 2006; Vantaux et al. 2011).

La preferencia entre fuentes de alimento que conduce a un mayor reclutamiento necesariamente involucra la cantidad secretada de la feromona reclutadora. El tiempo bebiendo y el volumen ingerido se propone como mecanismo en la decisión de secretar feromona reclutadora en hormigas que atienden áfidos (Mailleux et al. 2000). Para Pheidole bilimeki se descarta ese mecanismo ya que no se observaron diferencias en los tiempos de beber ante cada uno de los carbohidratos. Además, la poca variación en el tiempo de beber sugiere que esta hormiga parece que bebe un volumen en función de la capacidad del estómago social, más que en función del tipo de químico. Los tiempos bebiendo se pueden incrementar cuando se duplica la concentración de sacarosa a través de un fenómeno que depende del aumento de la viscosidad de la solución al interferir con la fluidez en el proceso de la ingesta (Josens et al. 1998).

La hormiga Pheidole bilimeki es una especie generalista común en hábitats con disturbio y frecuentemente dominante en zonas urbanas (Longino \& Cox 2009). Esto es consistente con el hecho de presentar respuesta de reclutamiento, dependiente de la concentración, a todos los carbohidratos probados y que son comunes en plantas, 
secreciones de áfidos y hemolinfa de insectos. No obstante su amplio espectro en la explotación de recursos, se demuestra que presenta claras preferencias entre azúcares sobre todo cuando existe la alternativa de elección. Se puede asumir bajo el principio de parsimonia de la naturaleza que la elección implica el mejor balance esfuerzo-beneficio. Es decir, se reclutan más individuos hacia el recurso de más alto valor energético a través de una mayor secreción de hormona reclutadora, un proceso que podría estar determinado por la interacción cuantitativa o cualitativa del tipo de carbohidrato con su receptor en las estructuras bucales, como ya ha sido estudiado en la mosca de la fruta (Freeman et al. 2014). Los recientes avances en la secuenciación del genoma en especies de hormigas (Smith et al. 2011; Wurm et al. 2011; Roux et al. 2014), abre un amplio panorama para comprender los mecanismos moleculares que subyacen en sus distintas capacidades adaptativas para la explotación eficiente de los recursos disponibles.

AGRADECIMIENTOS. Al Consejo Nacional de Ciencia y Tecnología por el apoyo a través de becas de posgrado (321229). Al Tecnológico Nacional de México (antes Dirección General de Educación Superior Tecnológica) por los apoyos a través de proyectos de investigación. Al Dr. John Longino (Utah Museum of Natural History) por la corroboración taxonómica de la especie. Asimismo, a los revisores anónimos por sus acertadas sugerencias al manuscrito.

\section{LITERATURA CITADA}

Ayre, G. L. 1967. The relationships between food and digestive enzymes in five species of ants (Hymenoptera: Formicidae). Canadian Entomologist, 99: 08-411.

Becker, A., Schlöder, P., Steele, J. E. \& Wegener, G. 1996. The regulation of trehalose metabolism in insects. Experientia, 52: 433-439.

Blüthgen, N. \& Fiedler, K. 2004. Preferences for sugars and amino acids and their conditionality in a diverse nectar-feeding ant community. Journal of Animal Ecology, 73: 155-166.

Blüthgen, N., Gottsberger, G. \& Fiedler, K. 2004. Sugar and amino acid composition of ant-attended nectar and honeydew sources from an Australian rainforest. Austral Ecology, 29: 418-429.

Breed, M. D., Fewell, J. H., Moore, A. J. \& Williams, K. R. 1987. Graded recruitment in a ponerine ant. Behavioural Ecology and Sociobiology, 20: 407-411.

Cammaerts, M. \& Cammaerts, C. R. 1980. Food recruitment strategies of the ants Myrmica sabuleti and Myrmica ruginodis. Behavioural Processes, 5: 251-70.

Carthy, J. D. 1950. Odour trails of Acanthomyops fuliginosus. Nature, 166: 154.

Comisión Nacional del Agua (CNA). Servicio Meteorológico Nacional, México, 2014. Recurso electrónico: http://smn.cna.gob. mx/index.php?option=com_content\&view=article\&id=166\&tmp $\mathrm{l}=\mathrm{com}$.

Detrain, C. \& Deneubourg, J. L. 1997. Scavenging by Pheidole pallidula: a key for understanding decision-making systems in ants. Animal Behavioural, 53: 537-547.
Detrain, C. \& Prieur, J. 2014. Sensitivity and feeding efficiency of the black garden ant Lasius niger to sugar resources. Journal of Insect Physiology, 64: 74-80.

Douglas, A. E. 2003. The nutritional physiology of aphids. Advances in Insect Physiology, 31: 73-40.

Douglas, A. E. 2006. Phloem-sap feeding by animals: problems and solutions. Journal of Experimental Botany, 57: 747-754.

Freeman, E. G., Wisotsky, Z. \& Dahanukar, A. 2014. Detection of sweet tast ants by a conserved group of insect gustatory receptors. Proceedings of the National Academy of Sciences U.S.A, 111: 1598-1603.

Friedman, S. 1985. Carbohydrate metabolism, pp. 43-76. In: L. I. Gilbert and G. A. Kerkut (Eds.). Comprehensive Insect Physiology, Biochemistry and Pharmacology. Pergamon Press, Oxford.

Heil, M., Rattke, J. \& Boland, W. 2005. Postsecretory hydrolysis of nectar sucrose and specialization in ant/plant mutualism. Science, 308: 560-563.

Hendrix, D. L., Wei, Y. A. \& Leggett, J. E. 1992. Homopteran honeydew sugar composition is determined by both the insect and plant species. Comparative Biochemistry and Physiology B: Biochemistry and Molecular Biology, 101: 23-27.

Hölldobler, B. \& Wilson, E. O. 1990. The ants. The Belknap Press of Harvard University Press. Cambridge, Massachusetts. 732 pp.

Horta-Vega, J. V., Eguía-López, B., Ruiz-Cancino, E. \& BrussoloCeballos, R. 2010. Recruitment response to six carbohydrates in the ant Pheidole dentigula Smith, 1927 (Hymenoptera: Formicidae). Entomological News, 121: 290-297.

Josens, R. B., Farina, W. M. \& Roces, F. 1998. Nectar feeding by the ant Camponotus mus: intake rate and crop filling as a function of sucrose concentration. Journal of Insect Physiology, 44: 579-585.

Kay, A. 2004. The relative availabilities of complementary resources affect the feeding preferences of ant colonies. Behavioral Ecology, 15: 63-70.

Kiss, A. 1981. Melezitose, aphids and ants. Oikos, 37: 382.

Koptur, S. \& Truong, N. 1998. Nectar sugar preferences of introduced pest ant species in South Florida. Biotropica, 30: 179-189.

Longino, J. T. \& Cox, D. J. 2009. Pheidole bilimeki reconsidered (Hymenoptera: Formicidae). Zootaxa, 1985: 34-42.

Mailleux, A. C., Deneubourg, J. L. \& Detrain, C. 2000. How do ants assess food volume?. Animal Behaviour, 59: 1061-1069.

Ricks, B. L. \& Vinson, S. B. 1970. Feeding acceptability of certain insects and various water-soluble compounds to two varieties of the imported fire ant. Journal of Economic Entomology, 63: 145-148.

Ricks, B. L. \& Vinson, S. B. 1972. Digestive enzymes of the imported fire ant, Solenopsis richteri (Hymenoptera: Formicidae). Entomologia Experimentalis et Applicata, 15: 329-334.

Roux J., Privman, E., Moretti, S., Daub, J. T., Robinson-Rechavi, M. \& Keller, L. 2014. Patterns of positive selection in seven ant genomes. Molecular Biology and Evolution, 31: 1661-1685.

Smith, C. D., Zimin, A., Holt, C., Abouheif, E., Benton, R., Cash, E., Croset, V., Currie, C. R., Elhaik, E., Elsik, C. G., Fave, M. J., Fernandes, V., Gadau, J., Gibson, J. D., Graur, D., Grubbs, K. J., Hagen, D. E., Helmkampf, M., Holley, J. A., Hu, H., Ibarraran-Viniegra, A. S., Johnson, B. R., Johnson, R. M., Khila, A., Kim, J. W., Laird, J., Mathis, K. A., Moeller, J. A., Muñoz-Torres, M. C., Murphy, M. C., Nakamura, R., Nigam, S., Overson, R. P., Placek, J. E., Rajakumar, R., Reese, J. T., Robertson, H. M., Smith, C. R., Suarez,A. V., Suen, G., Suhr,E. L., Tao, S., Torres, C. W., van Wilgenburg, E., Viljakainen, L., Walden, K. K. O., Wild, A. L., Yandell, M., Yorke, J. A. \& Tsutsui,N. D. 2011. Draft genome of the globally 
widespread and invasive Argentine ant (Linepithema humile). Proceedings of the National Academy of Sciences U. S. A., 108: 56735678.

Tinti, J. M. \& Nofre, C. 2001. Responses of the ant Lasius niger to various compounds perceived as sweet in humans: a structure-activity relationship study. Chemical Senses, 26: 231-237.

Vander-Meer, R. K., Lofgren, C. S. \& Seawright, J. A. 1995. Specificity of the Red Imported Fire Ant (Hymenoptera: Formicidae) Phagostimulant Response to Carbohydrates. The Florida Entomologist, 78: 144-153.

Vantaux, A., Van den Ende, W., Billen, J. \& Wenseleers, T. 2011. Large interclone differences in melezitose secretion in the facultatively ant-tended black bean aphid Aphis fabae. Journal of Insect Physiology, 57: 1614-1621.

Völkl, W., Woodring, J., Fischer, M., Lorenz, M. W. \& Hoffmann, K. H. 1999. Ant-aphid mutualisms: the impact of honey-dew production and honeydew sugar composition on ant preferences. Oecologia, 118: 483-491.
Wilson, E. O. 1959. Source and possible nature of the odor trail of fire ants. Science, 129: 643-644.

Wilson, E. O. 1971. The Insect Societies. Cambridge University Press, Cambridge, UK. 548 pp.

Wilder, S. M. \& Eubanks, M. D. 2010. Extrafloral nectar content alters foraging preferences of a predatory ant. Biology Letters, 6: 177-179.

Wurm, Y., Wang, J., Riba-Grognuz, O., Corona, M., Nygaard, S., Hunt, B. G., Ingram, K. K., Falquet, L., Nipitwattanaphon, M., Gotzek, D., Dijkstra, M. B., Oettler, J., Comtesse, F., Shih, C. J., Wu, W. J., Yang, C. C., Thomas, J., Beaudoing, E., Pradervand, S., Flegel, V., Cook, E. D., Fabbretti, R., Stockinger, H., Long, L., Farmerie, W. G., Oakey, J., Boomsma, J. J., Pamilo, P., Yi, S. V., Heinze, J., Goodisman, M. A. D., Farinelli, L., Harshman, K., Hulo, N., Cerutti, L., Xenarios, I., Shoemaker, D. \& Keller, L. 2011. The genome of the fire ant Solenopsis invicta. Proceedings of the National Academy of Sciences U. S. A., 108: 5679-5684. 